Revista Ingeniería y Región. 2016;16(2): 49-55

http://dx.doi.org/10.25054/22161325.1298

\title{
Evaluación de tres alternativas de fertiriego en el establecimiento del cultivo de plátano (musa paradisiaca/harton cv) en el Yopal, Casanare
}

\author{
Evaluation of three alternatives of fertigation \\ in the establishment of platain crop (musa \\ paradisiaca/harton cv) in the Yopal, Casanare
}

Gustavo Castro G. ${ }^{1}$ y Huber P. Chiquillo ${ }^{2}$

\begin{abstract}
Resumen
En la actualidad es necesario implementar en la agricultura métodos de manejo racional del recurso hídrico. El cultivo de plátano requiere altos contenidos de agua y fertilizante. El fertiriego es una alternativa para suplir requerimientos hídricos y a la vez fertilizar la planta. La región de los llanos orientales se caracteriza por suelos franco-arcillosos, poco permeables y de velocidad de infiltración lenta. Lo que hace que la disposición de fertilizantes se localice en superficie del terreno, dificultando su paso hacia la zona radicular. En la presente investigación se evaluó en etapa de establecimiento tres alternativas de fertiriego (T1: subterráneo, T2: goteo, T3: drench). Se estableció un lote experimental compuesto por 30 unidades experimentales para cada tratamiento. La Lámina Rápidamente Aprovechable (LARA), se estableció teniendo en cuenta el Punto de Marchitez Permanente (PMP), la Capacidad de Campo (CC), la Profundidad Radicular (PR) y la densidad aparente (DA). Los requerimientos de fertilización se estimaron a partir de análisis de suelos. Las variables de respuesta fueron el número de hojas, diámetro del tallo, altura de la planta y peso de la planta. Para el ANOVA se utilizó un DCA y los datos fueron analizados en INFOSTAT mediante una prueba LSD Fisher con un valor $p$ valor de 0.05 . Se encontraron diferencias significativas entre los tratamientos, en donde el tratamiento de fertiriego subterráneo mostró mayores valores respecto a las variables de seguimiento. En este trabajo se encontró que la alternativa de fertiriego subterráneo podría ser una solución viable para un manejo eficiente del agua y fertilización en el cultivo de plátano, posiblemente a que esta alternativa dispone de manera local el agua y los nutrientes para la planta.
\end{abstract}

Palabras clave: musáceas; riego; fertirrigación.

1 Ing. Agrícola. M.Sc. Ing. Civil. Docente del programa de Ingeniería Agronómica Universidad de La Salle. Sede Utopía. El Yopal, Casanare.gcastrog@unisalle.edu.co.

2 Estudiante del programa de Ingeniería Agronómica. Integrante del Semillero de Investigación de las Musáceas-SIMUSA. Universidad de La Salle. Sede Utopía. El Yopal, Casanare. Colombia. hchiquillo44@unisalle.edu.co. 


\begin{abstract}
Currently, in agriculture it is necessary to implement methods of rational handling of water resource. Plantain cultivation required a high content of water and fertilizer. Fertigation is an alternative to supplying hydric requirements and at the same time fertilizing the plant. The region of Colombia's eastern plains is characterized by clay loam soils, little permeability and slow infiltration velocity. This makes the arrangement of fertilizers appear on the surface of the land thus making it difficult to move to the root zone. In the present research, three fertigation alternatives (T1: subterranean, T2: drip, T3: drench) were evaluated at the establishment stage. An experimental batch composed of 30 experimental units was established for each treatment.

The Readily Available Water RAW was obtained taking into account the Permanent Wilt Point (PMP), Field Capacity (CC), Root Depth (RD) and apparent density (DA). The requirement of fertilization was calculated from soil analysis. The response variables were the number of leaves, diameter of the stem and height and weight of the plant. For the ANOVA, a completely randomized design-CRD was used and data were analyzed by using an INFOSTAT LSD Fisher test with a $p$ value of 0.05 . Significant differences between the treatments were found, where the treatment of subterranean fertigation evidenced bigger values in relation to the follow-up variables. In this work, one observed that the alternative of subterranean fertigation could be an affordable solution for an efficient handling of water and fertilization in the cultivation of plantains due to the alternative arrangements of local water and nutrients for the plant.
\end{abstract}

Keywords: musáceas; irrigation; fertirrigation.

\section{Introducción}

La producción de plátano en Colombia es de gran importancia para la canasta familiar y la generación de empleo. Fisiológicamente la planta de plátano requiere de abundante cantidad de agua. Presentándose un aumento progresivo y variable en la necesidad del recurso hídrico a medida que la planta avanza en su crecimiento (Castaño, et al., 2012). Por esto un exceso o déficit en el contenido de humedad del suelo son un factor determinante en el desarrollo radicular de las plantas (Guzmán, 2010), lo que repercute en el crecimiento y en la producción al limitarse la absorción de nutrientes (Soto, 1990).

En la producción de plátano los efectos por exceso o deficiencia del agua o fertilizante son notorios y se ven reflejados en el desarrollo de la planta y en la producción (Hernández, et al., 2007). Aparte que la respuesta de las plantas a la fertilización no es uniforme en todos los suelos, ya que depende de un contenido inicial de nutrientes (Furcal \& Baquero, 2013), y el cultivo de plátano requiere de altas dosificaciones de fertilizante, especialmente $\mathrm{K}^{+}$(Labarca, et al., 2005). Por esto se debe definir las dosificaciones de fertilización en relación al contenido de nutrientes en el suelo, y suministrar los contenidos de agua para una condición de suelo especifica. El fertiriego es una tecnología que combina la aplicación de agua y fertilizantes, permitiendo el riego localizado de alta frecuencia, y un bulbo húmedo con nutrientes que se concentra en el área radicular (Rodríguez \& Puig, 2012); (Casanova \& Rivero, 2006). Realizando aplicaciones de fertirriego que satisfagan las necesidades del cultivo dependiendo de su estado fenológico (Camejo, et al., 2010).

Debido a la problemática de la región en cuanto a sus características de suelo franco-arcillosos, poco permeables y de velocidad de infiltración lenta, que dificulta la eficiencia en la aplicación de del agua y fertilizantes en diferentes cultivos, generando una baja productividad. Se implementaron tres alternativas de fertirriego para el cultivo de plátano en sus etapas iniciales.

\section{Materiales y métodos}

\subsection{Ubicación del experimento}

El proceso investigativo se llevó a cabo en el centro de investigaciones agrícolas y pecuarias San José Matepantano de la Universidad de la Salle en el campus del programa de ingeniería agronómica, proyecto Utopía, Ubicado a 12 kilómetros de la zona urbana del municipio de El Yopal departamento de Casanare, en las coordenadas 5 $5^{\circ} 19^{\prime} 18.75^{\prime \prime} \mathrm{N}$, $72^{\circ} 17^{\prime} 01,4$ ' $\mathrm{O}$, en el lote correspondiente al agro- 
ecosistema musáceas, a una altitud de 248 msnm (bosque húmedo tropical). Precipitación de 1500 a 2500 $\mathrm{mm}$ anuales, con un promedio de $2000 \mathrm{~mm}$ al año, con temperaturas entre 20 y $36^{\circ} \mathrm{C}$ con promedios de $27^{\circ} \mathrm{C}$.

En campo se establecieron tres tratamientos con 10 réplicas, para un total de treinta unidades experimentales. El sistema de siembra fue en cuadro, con $1 \mathrm{~m}$ entre plantas y $2 \mathrm{~m}$ entre surcos. Los tratamientos fueron T1 subterráneo, T2 goteo y el T3 drench. Las variables a evaluar fueron el número de hojas, el diámetro del tallo, altura de la planta y peso de la planta. Para esta investigación se realizó un diseño experimental completamente al azar (DCA), el análisis de los datos se realizó en el sofware INFOSTAT mediante una prueba LSD Fisher con un nivel de significancia de 0.05 .

\subsection{Manejo del cultivo}

Para la implementación del cultivo se preparó el terreno con 2 pases de arado de discos, en un área de 207 $\mathrm{m}^{2}$ previo al establecimiento de las plantas se realizó el ahoyado, encalado y la selección de cormos entre 500 gr y 1000 g, la desinfección se realizó de forma localizada en el sitio de siembra, combinando un insecticida (clorfluazuron) y un fungicida (mancozeb). El procedimiento a seguir incluye control de enfermedades, con rotación de moléculas, control mecánico de arvenses, monitoreo y control manual de plagas, fertilizaciones foliares, deshoje, deshije y aporque una vez al mes a partir del segundo mes de establecido en cultivo.

\subsection{Instalación de los sistemas de riego}

Una vez establecidas las plantas se procede con la instalación de los sistemas de riego, iniciando con la instalación de un tanque de 1.000 litros, en donde se prepara la solución, ubicado a una altura de 1,6 m. De la tubería principal se desprenden dos secundarias una de $1 / 2$ " correspondiente al fertirriego subterráneo de la cual desprenden tres ramales de $10 \mathrm{~m}$ de longitud, con manguera de $1 / 2$ " a la cual se le realizaron los goteros con un taladro en dirección a cada planta, enterrándose a $20 \mathrm{~cm}$ de profundidad. El otro ramal es de 2", correspondiendo al fertirriego por goteo, desprendiéndose también tres ramales de $10 \mathrm{~m}$ de longitud, con cintas de goteo superficial, a estos sistema se les determino el caudal utilizando una probeta y un cronometro. El riego en drench se realiza a balde, preparando la solución de sales en un tanque de 200 litros ubicado al lado del lote.

\subsection{Dosificación de sales de fertirriego}

Las dosificaciones de las sales de fertirriego se determinaron en base a un análisis de suelo realizado en el laboratorio de suelos de la universidad de La Salle campus Utopía, para esto se tomaron 10 submuestras a $20 \mathrm{~cm}$ de profundidad en todo el lote, para combinarlas y formar una muestra para secarla a temperatura ambiente. Las sales utilizadas son nitrato de amonio, ácido fosfórico y cloruro de potasio, se procedió con las aplicaciones de fertirriego parciales cada 8 días. El caudal del gotero (T1) fue de $0,31 / \mathrm{h}$, el caudal del sistema subterráneo (T2) de 4 1/h y el sistema en drench (aplicación directa) (T3), se realizó con un balde.

\subsection{Estimación de la lámina rápidamente aprovechable-LARA}

La muestra de suelo a partir de la cual se determina la capacidad de campo CC, el punto de marchitez permanente PMP y la densidad aparente Da, fue extraída con el barreno y los anillos de volumen conocido, para este caso se tomaron cuatro muestras a $20 \mathrm{~cm}$ de profundidad.

La humedad gravimétrica se determinó secando la muestra en horno a $105^{\circ} \mathrm{C}$ durante 24 horas (Singer \& Munns, 1999), obteniendo su valor mediante la siguiente expresión(1).

$$
W=\frac{P s h-P s s}{P s S} * 100
$$

La capacidad de campo se obtuvo mediante el método de mesa de tensión sandbox Model 08.01, (Eijkelkamp Co., Ltd., Holanda). a ecuación 2 muestra la ecuación tenida en cuenta para el calculo del punto de marchitez permanente (PMP) (Silva, et al., 1988).

$$
\% \mathrm{PMP}=-5+0,74 * \% \mathrm{CC}
$$

Para calcular la densidad aparente se relaciona el peso seco del suelo con el volumen total del cilindro (Ecuación 3).

$$
D a=\frac{P S S}{V t}
$$

Donde:

W: Humedad gravimétrica (\%)

Psh; Peso del suelo húmedo (g) 
Pss: Peso del suelo seco (g)

\%PMP: Punto de marchitez permanente (\%)

$\%$ CC: Capacidad de campo (\%)

Da: Densidad aparente $\left(\mathrm{g} / \mathrm{cm}^{3}\right)$

Vt: Volumen total

Para finalmente determinar la lámina de agua aprovechable ecuación (4).

$$
L A R A=\frac{C C-P M P}{100} * D a * P r * 10
$$

Donde:

LARA: Lámina de agua aprovechable (mm)

PMP: Punto de marchitez permanente (\%)

CC: Capacidad de campo (\%)

Da: Densidad aparente $(\mathrm{g} / \mathrm{cm} 3)$

Pr: profundidad radicular $(\mathrm{cm})$

\subsection{Seguimiento de variables de respuesta}

Se realizó mediciones de las variables de diámetro, altura y número de hojas. La variable de peso de la planta o biomasa es subdividida en dos partes longitud de raíces y peso total de la planta. Para esto fueron extraídas tres plantas por cada tratamiento, se les retiró la tierra, para llevarla al laboratorio de la Universidad de La Salle campus Utopía, en donde se midió la longitud y el peso total de la planta en fresco. Posteriormente se llevó el material vegetal al horno hasta deshidratar completamente la planta, una vez secas se toma nuevamente el peso total de la planta, para obtener una relación en porcentaje de materia seca, con la expresión (5).

$$
\text { Biomasa }=\frac{P f-P s}{P f} * 100
$$

Donde:

Ps: peso seco.

Pf: peso fresco.

\section{Resultados y discusión}

La figura 1 muestra los resultados del análisis de suelos considerado para la ejecución del proyecto, se aprecian niveles altos de aluminio y hierro, ideal para el calcio y bajo para los demás elementos. El plan de fertirriego se desarrolló en base a estos resultados se-

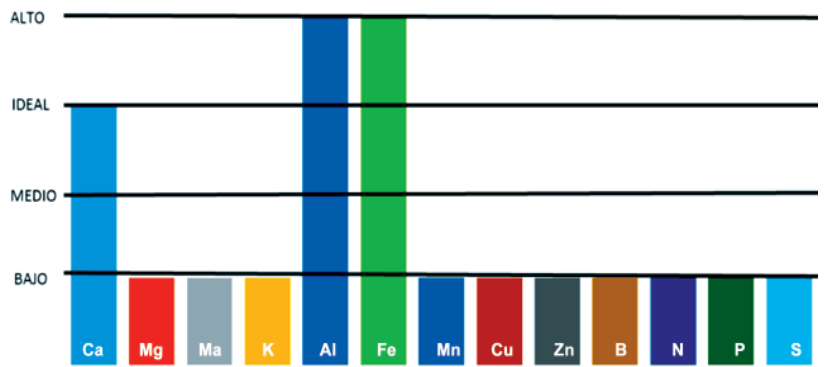

Figura 1. Interpretación de los resultados del análisis de suelos.

gún los requerimientos nutricionales del plátano (Lardizabal, 2007). Se utilizaron $1 \mathrm{~m}^{3}$ de riego parcial para cada tratamiento. Las dosis de fertilizantes de $513 \mathrm{ml} / \mathrm{m}^{3}$ de nitrato de amonio, $826 \mathrm{gr} / \mathrm{m}^{3}$ de ácido fosfórico y $219 \mathrm{gr} / \mathrm{m}^{3}$ de cloruro de potasio.

La siguiente Tabla 1 muestra los valores obtenidos en la caracterización de las propiedades utilizadas en el estudio. La LARA fue estimada en un valor de 33.6 $\mathrm{mm}$, es decir 33.6 litros $/ \mathrm{m}^{2}$, lo que se entiende como el volumen aplicado de riego por planta.

La figura 2 muestra el comportamiento de los resultados respecto al número de hojas, se aprecia que estadísticamente no se encontraron diferencias significativas, sin embargo el T1 generó valor. La emisión foliar en plátano es constante en sus primeras etapas (Aristizábal, 2008), esta tiene una relación directa con el desarrollo radicular y la altura de la planta (Aristizábal \& Jaramillo, 2010). En la etapa inicial la planta se enfoca en el desarrollo radicular y la tasa de emisión es constante con el crecimiento del pseudotallo, desarrollando hojas más grandes a medida que la planta crece (Martínez \& Cayón, 2011), indicando que el número

Tabla 1. Caracterización de parámetros considerados en el estudio.

\begin{tabular}{l|c|c}
\hline \multicolumn{1}{c|}{ Parámetro } & Unidades & Valor \\
\hline Capacidad de campo (CC) & $\%$ & 22,98 \\
\hline Densidad aparente (Da) & $\mathrm{gr} / \mathrm{cm}^{3}$ & 1,6 \\
\hline $\begin{array}{l}\text { Punto de Marchitez } \\
\text { Permanente (PMP) }\end{array}$ & $\%$ & 12,48 \\
\hline LARA $^{*}$ & $\mathrm{Mm}$ & $33,6 \mathrm{~mm}$. \\
\hline Arena & $\%$ & 45,88 \\
\hline Arcilla & $\%$ & 33,75 \\
\hline Limo & $\%$ & 20,38 \\
\hline Textura & & $\begin{array}{c}\text { Franco Arcillo } \\
\text { Arenoso }\end{array}$ \\
\hline
\end{tabular}

${ }^{*}$ El valor utilizado de PR profundidad radicular: $20 \mathrm{~cm}$. 


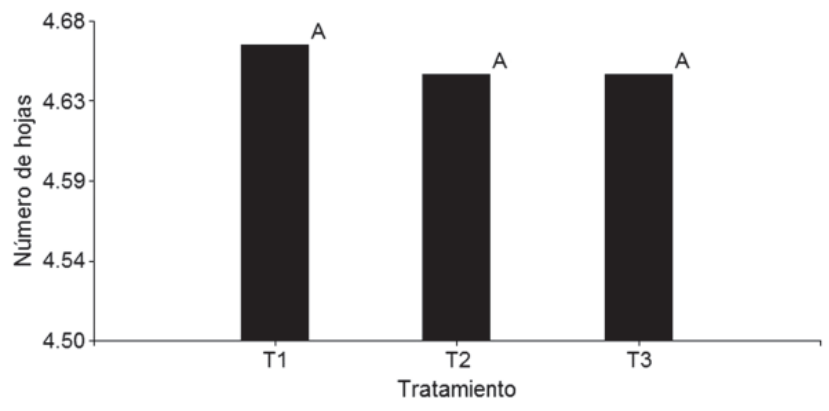

T1: subterráneo. T2: goteo: T3: drench

Figura 2. Número de hojas con respecto a los tres sistemas de fertirriego.

de hojas funcionales que las plantas de plátano poseen en cada etapa es constante, principalmente en sus primeras etapas y aumenta en número a medida que la planta crece.

La figura 3 muestra el comportamiento respecto a la altura de la planta. Se observó que el T1 presentó la mayor altura de la planta. Se aprecia el efecto entre el método de fertiriego subterráneo y el crecimiento de la planta. El agua y los nutrientes dispuestos a la profundidad de $20 \mathrm{~cm}$ (directamente en la zona radicular), influyen positivamente en el crecimiento de la misma (Barrera, et al., 2011); (Furcal \& Baquero, 2014).

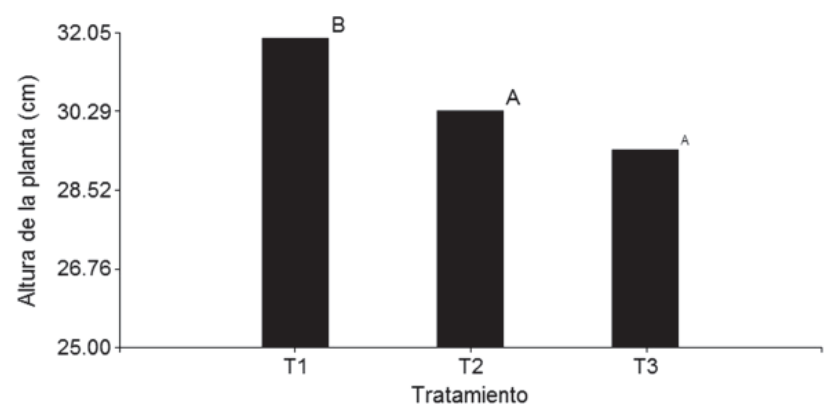

T1: subterráneo. T2: goteo: T3: drench

Figura 3. Altura de la planta respecto a los métodos de fertirriego.

La Figura 4 muestra los resultados respecto al diámetro del pseudotallo, se encontraron diferencias significativas entre los tratamientos. Castro \& Sosa (2015) en un experimento de fertilización en plátano, encontraron que las plantas que alcanzaron mayor altura, tenían la característica de tener un pseudotallo menor, sin embargo, los tratamientos que generaron mayor cantidad de raíces fueron los tratamientos con diámetros de cormo mayores y menor altura de la planta.

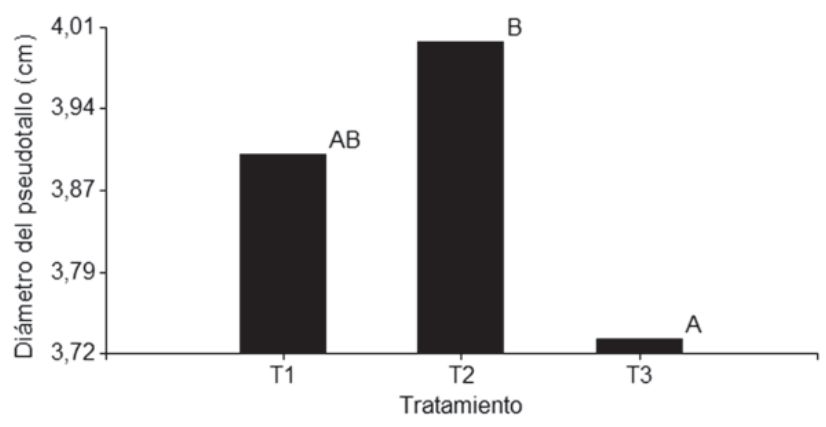

T1: subterráneo. T2: goteo: T3: drench

Figura 4. Diámetro del pseudotallo respecto a los métodos de fertirriego.

La figura 5 muestra la respuesta de las raíces a los sistemas evaluados. No se encontraron diferencias significativas entre los tratamientos, en el momento de realizar el muestreo se observó que en el sistema subterráneo (T1) las raíces se dirigían hacia los costados y hacia abajo, concentrándose en mayor porcentaje desde los 0 a los $30 \mathrm{~cm}$, concordando con lo mencionado por Martínez \& Rodríguez (2015) los cuales han informado que el $90 \%$ de la masa radicular del plátano se encuentra en los primeros $40 \mathrm{~cm}$ de profundidad. Para T2 las raíces se concentraron cerca del cormo. Rodríguez et al (2005) establecen que un sistema de riego por goteo, induce el desarrollo superficial del sistema radical de la planta, el cual puede producir pérdidas por volcamiento en las plantas. Esta situación podría verse presente en los suelos estudiados debido a que la textura franca areno arcillosa hace que la planta tenga dificultades para que su sistema radicular se desarrolle adecuadamente. Se ha evidenciado que el riego por goteo podría convertirse en una adecuada alternativa para el cultivo de plátano (Rodríguez, et al., 2003), sin embargo debe considerarse el tipo de textura del suelo que permita un buen desarrollo

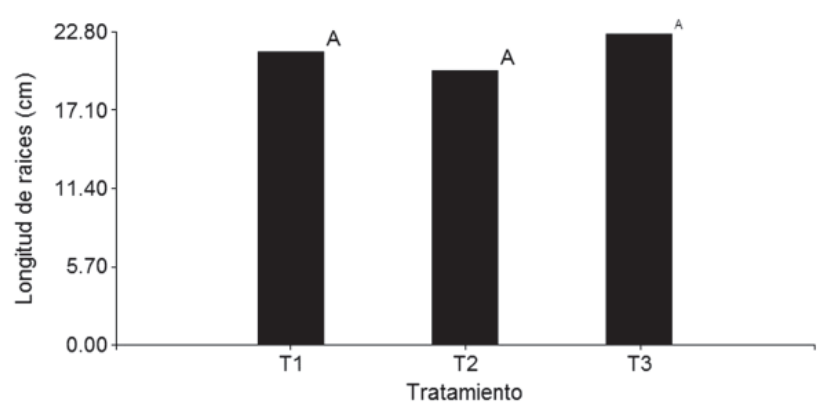

T1: subterráneo. T2: goteo: T3: drench

Figura 5. Longitud de raíces respecto a los métodos de fertirriego. 
radicular. E1 T3 mostró que el desarrollo radicular se extiende hacia los costados del cormo y busca la superficie del terreno.

La figura 6 muestra la relación entre peso fresco y peso seco de los tratamientos. El T1 presentó los mayores resultados, estableciendo según el análisis estadístico diferencias significativas. Según Castillo et al (2011) las primeras etapas del cultivo de plátano son críticas en cuanto la acumulación de nutrientes, para el posterior desarrollo de la planta y producción de la misma. Con esto se define la influencia que tiene la fertilización y la forma como se aplica con respecto a la acumulación de materia seca por parte de la planta. Martínez \& Cayón (2011) resaltan el proceso de acumulación de materia seca en la planta en su proceso de desarrollo, estableciendo que inicialmente existe una mayor acumulación en el cormo, permitiendo un posterior desarrollo de la parte área de la planta. Así se recalca que el crecimiento y desarrollo de la planta de plátano es influenciada por las condiciones naturales en que es establecida y las condiciones que se le da a la misma en cuanto a suministro de agua y fertilizantes.

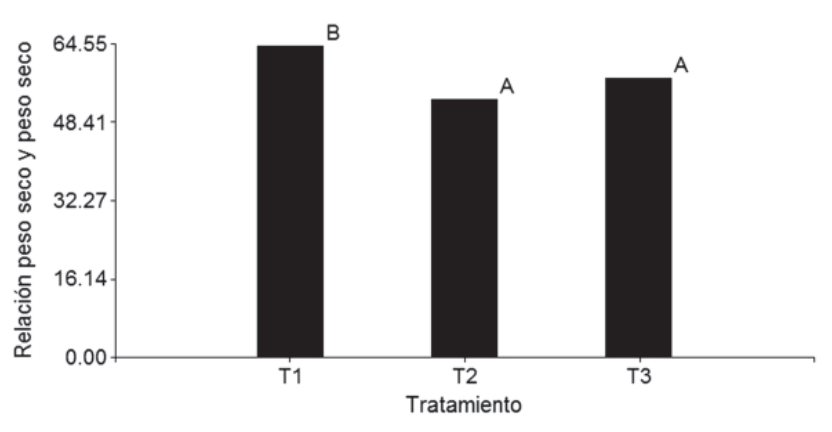

T1: subterráneo. T2: goteo: T3: drench

Figura 6. Relación peso seco y peso fresco respecto al sistema de fertirriego.

\section{Conclusión}

Se evidenció la respuesta en la etapa de establecimiento de los tres métodos de fertiriego estudiados. El sistema subterráneo de fertiriego permitió corroborar que al colocar el agua y los nutrientes para este tipo de suelo de textura franca-arcillo-arenosa a la profundidad radicular, es posible generar un estímulo positivo en el desarrollo fisiológico de la planta. Se apreció como el sistema por goteo estimula el desarrollo de la planta, sin embargo, las raíces tienden a buscar la superficie, lo que hace necesario realizar labores de aporque y prevención de volcamiento de las plantas. El desempeño del tratamiento en dench fue muy similar al tratamiento de riego por goteo.

\section{Agradecimientos}

A la universidad de La Salle, a la facultad de ciencias agropecuarias, al programa de ingeniería agronómica sede Utopía, Al Ing Ricardo Bueno director de programa, a los estudiantes de la línea productiva Lizeth Díaz, Olga Medina Sánchez, Linconl Barragan Buitrago, Dainer Pama Pianda, Jimmy Rojas Bonilla, Uriel Gonzalez Jiménez, Gerson Andrés Aguilar Godoy, Zulman Eulises Castillo Caicedo, Julián Andres Cuevas Rayo, Luis Fernando Cardona Grajales.

\section{Referencias bibliográficas}

Aristizábal, L. M., 2008. Evaluación del crecimiento y desarrollo foliar del plátano hondureño enano (Musa $\mathrm{AAB})$ en una región cafetera colombiana. Agron., 16(2):23-30. ISSN 0568-3076.

Aristizábal, L. M., Jaramillo, G. C., 2010. Identificación y descripción de las etapas de crecimiento del plátano dominico hartón (Musa AAB). Agron 18(1):2940. ISSN 0568-3076.

Barrera, J. L., Combatt, E. M., Ramírez, Y. L., 2011. Efecto de abono orgánico sobre el crecimiento y producción del plátano Hartón (Musa AAB). Revista colombiana de ciencias hortícolas. 5(2):186-194.

Camejo, L. E., Duarte, L. S., Companioni, J. L., Paneque, P. 2010. Tecnología de riego y fertirrigación en ambientes controlados. Revista Ciencias Técnicas Agropecuarias, 19(1):95-97. ISSN 2071-0054.

Casanova, O. E., Rivero, C., 2006. Efecto de fuentes alternativas de fertilizantes con el método de la fertirrigación sobre la nutrición mineral y rendimientos de bananos en una finca del estado Aragua, Venezuela. Agronomía Tropical, 56(3):325-344. ISSN 0002-192X.

Castaño, Á. M., Aristizábal, M., González, H., 2012. Requerimientos hídricos del plátano dominico- hartón (Musa AAB Simmonds) en la región santágueda (Palestina, Caldas). Revista de UDCA Actualidad y Divulgación Científica, 15(2):331-338. ISSN 01234226. 
Castillo, G. A. M., Hernández, M. J. A., Avitia, G. E., Pineda, P. J., Valdéz, A. L. A., Corona, T. T., 2011. Extracción de macronutrientes en banano 'Dominico' (Musa spp.). Phyton (Buenos Aires), 80(1):65-72. ISSN 1851-5657.

Castro, G. G., Sosa, R. M. D., 2015. Propagación de hijuelos de plátano (Musa paradisiaca/Hartón cv) en El Yopal, Casanare. Revista SEMICIEBB, Vol. 1, No. 1. ISSN 2500-4662.

Furcal, B. P., Barquero, B. A., 2013. Respuesta del plátano a la fertilización con $\mathrm{P}, \mathrm{K}$ y $\mathrm{S}$ durante el primer ciclo productivo. Agronomía Mesoamericana, 24(2): 317-327. ISSN 1021-7444.

Furcal, B. P., Barquero, B. A., 2014. Fertilización del plátano con nitrógeno y potasio durante el primer ciclo productivo. Agronomía Mesoamericana, 25(2): 267-278. ISSN 2215-3608.

Hernández, Y., Marín, M., y García, J., 2007. Respuesta en el rendimiento del plátano (Musa AAB cv. Hartón) en función de la nutrición mineral y su ciclo fenológico. Parte I. Crecimiento y producción. Revista de la Facultad de Agronomía, 24(4):607-626. ISSN 0378-7818.

Labarca, M., Sosa, L., Esparza, D., Nava, C., Fernandez, L., Villar, A. 2005. Evaluación de la colocación del fertilizante en la planta madre una vez cosechada sobre las variables de crecimiento y producción en el cultivo del plátano Harton (Musa AAB). Revista de la Facultad de Agronomía, 22(4):416-428. ISSN 0378-7818.

Lardizabal, R., 2007, Producción de plátano de alta densidad, Cuenta del Desafío del Milenio de Honduras. MCA-Honduras, La lima. Cortes. Honduras, p. 35
Martínez, A. A. M., Cayón, S. D. G., 2011. Dinámica del Crecimiento y desarrollo del Banano (Musa AAA Simmonds cvs. Gran Enano y Valery). Revista Facultad Nacional de Agronomía, 64(2):6055-6064.

Martínez, V. R., Rodríguez, G. M. R., 2015. Respuesta económica del cultivo de banano al riego por goteo subterráneo. Revista Ingeniería Agrícola, 5(1):27-33. ISSN 2306-1545.

Rodríguez, G. M. R., Puig, E. O., 2012. Comportamiento hidráulico de los sistemas de riego por goteo superficial y sub superficial. Revista Científicas Técnicas Agropecuarias, 21(3):23-28. ISSN 1010-2760.

Rodríguez, M., Rey, R., Sarmiento, O., 2005. Influencia del riego por goteo superficial y subterráneo sobre la distribución radical dl banano. Revista Científicas Técnicas Agropecuarias, 14(2):44-48. ISSN 1010-2760.

Rodríguez, M. R., Rey, R., Torralba, V., Sarmiento, O., 2003. Comportamiento del patrón de mojado bajo riego por goteo subsuperficial y superficial, 1er Congreso Internacional de Riego y Drenaje. Cuba Riego 2003, ISBN: 959-7164-49-3.

Silva, A., Ponce De León, J., García, F., Durán, A., 1988. Aspectos Metodológicos en la determinación de la Capacidad de ALEJAR Agua de Los Suelos del Uruguay. Facultad de Agronomía, Boletín de Investigación, $\mathrm{N}^{\circ} 10$.

Singer, M., Munns, D. 1999. Soil an Introduction. Cuarta Edición. Prentice Hall. USA, p. 527.

Soto, M., 1990. Bananos, cultivo y comercialización. Ministerio de agricultura y ganadería. San José. Costa Rica. ISBN: 997747057X, 9789977470573. 627p.[* | En linea. WMF *] 
